\title{
Síndrome de Burnout entre hombres y mujeres medido por el clima y la satisfacción laboral ${ }^{1}$
}

\author{
Mauricio Ramírez Pérez \\ Universidad de Tarapacá, Arica, Chile. \\ Email: mramirezp@achs.cl \\ Sau-Lyn Lee Maturana \\ Universidad de Tarapacá, Arica, Chile. \\ Email: slee@uta.cl
}

Resumen: El presente estudio busca determinar posibles diferencias por sexo en la aparición de variables asociadas al síndrome de Burnout medidos a través del clima y la satisfacción organizacional en una muestra de 233 trabajadores de la ciudad de Arica, pertenecientes a cinco diferentes actividades económicas (educación, salud, comercio de tangibles e intangibles, y administración pública).

Palabras Claves: Síndrome de Burnout, Clima Organizacional, Satisfacción Laboral, Factores Psicosociales.

\section{Burnout's syndrome in men and women measured by the job climate and job satisfaction}

Abstract: This study aims to determine possible gender differences in the occurrence of variables associated with Burnout syndrome as measured by the organizational climate and job satisfaction, in a sample of 233 workers from the city of Arica, belonging to five different economic activities (education, health, trade in tangible and intangible, and public administration).

Key Words: Burnout's syndrome, Organizational Climate, Job Satisfaction, Psychosocial Factors.

\section{Síndrome de Burnout entre os homens e mulheres medida pela clima e satisfação do trabalho}

Resumo: Este estudo procurou determinar a possível diferenças de sexo em a ocorrência de variáveis associadas com a síndrome de burnout, medida pelo clima e satisfação organizacional em uma amostra de 233 trabalhadores da cidade de Arica, pertencentes a cinco diferentes actividades económicas (educação , saúde, comércio de administração de materiais e imateriais, e público).

Palavras-chave: Síndrome de Burnout, Clima Organizacional, Satisfação no trabalho, fatores psicossociais. 


\section{Introducción}

La actividad laboral es una de las múltiples actividades que el hombre desarrolla a lo largo de su vida, ahora bien, esta interacción social que se produce entre el trabajador y su entorno, puede tener resultados beneficiosos y pueden llegar a ser causantes de importantes trastornos en la actividad laboral, no sólo afectando de forma individual, sino también en términos organizacionales y sociales.

En sus inicios, las tradiciones griegas y judeo-cristianas relacionaban el trabajo con actividades asociadas al castigo del ser humano, sin embargo, con el tiempo, estas actividades comenzaron a cambiar su connotación llegando a definir al hombre como tal, y vinculándolo incluso con la satisfacción de sus propias necesidades fundamentales (Gomes y Elizalde, 2009).

Actualmente, el trabajo es una de las actividades sobre las que se organizan las sociedades y por ello en los estados modernos se incluye entre las responsabilidades de los poderes públicos el velar por la salud, seguridad e higiene laboral (Peiró y Bravo, 1999). Bajo esta concepción Ramírez (2009), señala que es necesario propiciar trabajos que sean un aporte a la organización y la sociedad en general, pero además que la ejecución de este trabajo implique velar por la seguridad y el bienestar de los trabajadores, es decir, que no signifique un resultado negativo para la salud, tanto a un nivel físico como a un nivel psicológico.

Los cambios sociales y los cambios en la ejecución del trabajo, están reduciendo determinados riesgos al transformar los sistemas de trabajo y las actividades que las personas y equipos realizan, esto implica que emergen otros tipos de riesgos que no existían previamente. Tienden a reducirse determinados riesgos físicos pero aumentan los psíquicos y psicosociales (Peiró, 1999). Los factores psicosociales son definidos por el Instituto Nacional de Seguridad e Higiene en el Trabajo (INSHT) (1997), como aquellas condiciones que se encuentran presentes en una situación laboral y que están directamente relacionadas con la organización, el contenido del trabajo y la realización de la tarea, y que tienen capacidad para afectar tanto al bienestar o a la salud (física, psíquica o social) del trabajador como al desarrollo del trabajo. La preocupación por el estudio de estos riesgos psicosociales en el entorno laboral surge fundamentalmente por la alta relación encontrada entre factores psicosociales y enfermedades (Martín, Luceño, Jaén y Rubio, 2007).

En relación con esto, Chomali y Marjlurf (2007) señalan que las crecientes exigencias y expectativas de la persona y la cada vez mayor complejidad de nuestra sociedad hacen de las empresas un vehículo privilegiado para dar respuesta a las demandas y desafíos de nuestro tiempo. Fernández y Paravic (2003), señalan que el trabajo juega un papel destacado en la vida del hombre, dado que la mayoría de las personas pasan una tercera parte del día trabajando y lo hacen la mitad de su vida. De esto se despren- 
de la necesidad de establecer cuáles son las condiciones de trabajo, referido a los factores psicosociales que ponen en riesgo la salud de los trabajadores por el surgimiento del síndrome de Burnout, del mismo modo es preciso establecer las diferencias existentes entre hombres y mujeres.

\section{Síndrome de Burnout}

Se establece que el primer antecedente histórico del síndrome de Burnout se encuentra en el estudio realizado por Schwartz y Mill en 1953, quienes describían un sentimiento de baja moral y distanciamiento de los pacientes en enfermeras que trabajaban en un hospital psiquiátrico (Alarcón, Vaz y Guisado, 2002). Freudenberg en 1974 conceptualizó el Síndrome de Burnout como la sensación de agotamiento, decepción y pérdida de interés por la actividad laboral, que surge especialmente en aquellos que se dedican a profesiones de servicios como consecuencia del contacto diario con su trabajo (Ortega Ruiz y López Ríos, 2004).

En un principio este síndrome estaba adjudicado de forma exclusiva a aquellas profesiones que mantienen un contacto directo con sus beneficiarios - usuarios para el ejercicio de su actividad laboral (profesores, enfermeras, médicos, etc.), sin embargo Leiter y Schaufeli (1996); Demerouti, Bakker, De Jonge, Janssen y Schaufeli (2001); Gil-Monte (2003), han señalado que el síndrome de Quemarse por el trabajo (traducción realizada al Castellano), puede desarrollarse en profesionales cuyo trabajo no conlleve necesariamente el contacto directo con personas.

La definición del síndrome de Burnout más aceptada entre los investigadores, es la que ofrecen Maslach y Jackson (1986), quienes lo conceptualizaron como un síndrome de tres dimensiones: 1) agotamiento o cansancio emocional, definido como el cansancio y fatiga que puede manifestarse física y/o psíquicamente, con una sensación descrita como no poder dar más de si mismo a los demás; 2) despersonalización, como desarrollo de sentimientos, actitudes y respuestas negativas distantes y frías hacia otras personas, especialmente hacia los beneficiarios del propio trabajo; 3) por último, la baja realización personal o logro, que se caracteriza por una dolorosa desilusión para dar sentido a la propia vida y hacia los logros personales, con sentimientos de fracaso y baja autoestima (Maslach, Schaufeli y Leiter, 2001; Duran, Extremera, Montalbán y Rey, 2005; Sánchez, Guillen y León, 2006; Extremera, Durán y Rey, 2007).

De acuerdo a Italia, Favara-Scacco, Di Cataldo y Russo (2008), una de las principales características que presenta el síndrome es una reacción emocionalmente negativa activada en las personas, en respuesta a dificultades encontradas dentro del lugar de trabajo. De acuerdo a Ramírez y Zurita (2009) si estas respuestas negativas se enfrentan con un incremento en hábitos dañinos para la salud, eleva las posibilidades de presentar el Síndrome. 
Desde una perspectiva psicosocial, el síndrome de Quemarse por el trabajo se conceptualiza como una respuesta al estrés laboral crónico, que se caracteriza porque el individuo desarrolla una idea de fracaso profesional (en especial en relación a las personas hacia las que trabaja), la vivencia de encontrarse emocionalmente agotado, y actitudes negativas hacia las personas con las que trabaja (Gil-Monte y Peiró, 1999).

Para Gil-Monte y Peiró (2007), la necesidad de estudiar el síndrome de Quemarse por el trabajo viene unida a la necesidad de estudiar los procesos de estrés laboral, así como al reciente hincapié que las organizaciones han hecho sobre la necesidad de preocuparse más de la calidad de vida laboral que ofrecen a sus empleados. Existe evidencia que sugiere que las personas que presentan una visión más positiva hacia el trabajo que realizan, es decir, como carrera o como vocación, presentan menos probabilidad de padecer el síndrome de Burnout, mientras que los trabajadores que consideran su trabajo sólo como un trabajo, exhiben un mayor número de sujetos que presentan el Síndrome o están en riesgo de experimentarlo (Ramírez y Zurita, 2009).

\section{Clima Organizacional}

El Clima Organizacional es un concepto desarrollado inicialmente por Lewin, Lippitt y White (1939) al examinar la influencia de climas sociales creados experimentalmente sobre el comportamiento de niños en un grupo (Oliver, Tomas y Cheyne, 2005).

Desde esa primera aproximación se han desarrollado una serie de investigaciones, tal es así que para el Clima Organizacional se pueden identificar tantas definiciones como autores que han tratado de precisar el concepto. No existe un completo acuerdo entre las investigaciones en señalar un concepto único, sin embargo, predomina la definición que el Clima es el conjunto de percepciones que tienen sobre la organización los empleados de la misma considerados como un todo. Con independencia del autor o teoría de referencia, hay un amplio acuerdo en que el Clima Organizacional está compuesto por diferentes dimensiones que caracterizarían aspectos particulares del Ambiente Organizacional (Salgado, Remeseiro e Iglesias, 1996). Bajo esta perspectiva, es posible señalar que el Clima Organizacional es una representación socialmente construida y compartida respecto del ambiente laboral (Zohar y Luria, 2007).

A juicio de Lisbona, Palací y Gómez (2008), el Clima Organizacional es una propiedad del individuo que percibe la organización y es vista como una variable del sistema, que tiene la virtualidad de integrar al individuo, el grupo y la organización.

El Clima Organizacional ha sido considerado por la investigación psicosocial, una variable moduladora de los efectos de factores antecedentes como las prácticas de recursos humanos, la estructura organizacional, el 
liderazgo, etc. y determinadas variables consecuentes como la satisfacción laboral, el compromiso organizacional, la productividad, los conflictos, la ambigüedad de rol, etc. (Muñoz, Guerra de los Santos, Barón y Munduate, 2006). A juicio de González y Parera (2005), el Clima Organizacional adquiere especial interés por su influencia en los procesos organizacionales y psicológicos como la comunicación, toma de decisiones, solución de problemas, aprendizaje, motivación y, por ende, su influencia en la eficiencia de la organización y en la satisfacción de sus miembros. Esta visión es compartida por Laros y Puente-Palacios (2004), quienes señalan que la importancia de la investigación del Clima Organizacional es por la influencia que ejerce tanto en el comportamiento como sobre el desempeño de los trabajadores, pudiendo repercutir en la efectividad de las organizaciones.

Actualmente se debate sobre dos tipos de clima organizacional: el psicológico y el organizacional. El primero se estudia en el ámbito individual, mientras que el segundo se estudia en el ámbito organizacional. Ambos aspectos del clima son considerados fenómenos multidimensionales que describen la naturaleza de las percepciones que los empleados tienen de sus propias experiencias dentro de una organización. El tratamiento del clima como percepción genérica de situaciones ha tenido la ventaja de permitir evaluaciones sumarias del contexto en investigaciones que de otra manera estarían focalizadas en gran parte en el ámbito individual (Chiang, Salazar y Núñez, 2007).

\section{Satisfacción Laboral}

La satisfacción laboral es sin duda uno de los aspectos que los psicólogos de las organizaciones, tanto desde el punto de vista de la investigación como en el trabajo profesional, han prestado más atención (Meliá y Peiró, 1989; Dormán, Fay, Zapf, y Frese, 2006). Esto puede verse reflejado en lo señalado por Pozo, Alonso, Hernández y Martos (2005), quienes señalan que no existe una única ni unánime conceptualización del término. Apoyando a estos autores, para el desarrollo de esta investigación se encontraron 23.771 resultados para la búsqueda de Job Satisfaction limitando los años de búsqueda de 1999 a 2009 utilizando sólo la base de datos EBSCOhost,

Duran, et. al. (2005) señalan que el concepto de Satisfacción Laboral se ha constituido un habitual objeto de análisis en el ámbito psicosocial y podría ser definida como una respuesta afectiva o emocional positiva hacia el trabajo en general o hacia alguna faceta de éste. Diego, Diego y Olivar (2001) señalan que la Satisfacción Laboral es el resultado del cambio de demandas y las expectativas de una organización, y de las expectativas de los trabajadores y sus objetivos personales. Por lo que se puede señalar que entre los trabajadores y la organización debiesen existir relaciones de reciprocidad, esta reciprocidad se ve explicada cuando la organización proporciona los medios al trabajador para cubrir una serie de necesidades y realizar sus aspiraciones personales, y cuando la organización 
recibe por parte del trabajador (compromiso, dedicación, trabajo, etc.) para el cumplimiento de las metas organizacionales, por lo que se puede señalar que existe reciprocidad en el concepto.

En relación a lo anterior, Robbins (1999) señala que la Satisfacción Laboral es la diferencia entre la cantidad de recompensas que los trabajadores reciben y la cantidad que ellos creen que deberían recibir, esto representa una actitud más que un comportamiento. Este autor señala una serie de variables que determinan la Satisfacción Laboral, tales como trabajo mentalmente desafiante, recompensas justas, condiciones favorables de trabajo, apoyo de pares, compatibilidad entre la persona y el puesto, factores hereditarios. Del mismo modo señala el efecto de la Satisfacción Laboral en el trabajo, como mayor productividad, disminución del ausentismo y rotación laboral.

\section{Método}

Muestra: La muestra estuvo constituida por 233 trabajadores de la ciudad de Arica, pertenecientes a cinco diferentes actividades económicas: $30,5 \%$ son trabajadores de educación, 34,3\% corresponden a comercio de tangibles, un 17,2\% a trabajadores de ventas de intangibles, 9,9\% trabajadores de salud y 8,2\% a trabajadores de la administración pública.

Procedimiento: Para la recolección de datos se proporcionó a cada participante un cuadernillo con los instrumentos de medición asegurando la confidencialidad de los datos, sin embargo, de la muestra total sólo 159 de los cuestionarios fueron completados en su totalidad. De éstos un 64\% fueron mujeres y el 36\% hombres. Se utilizó un diseño no experimental de tipo descriptivo correlacional, ex post facto con variables categóricas e intervalares. Y el análisis de los datos se realizó a través del paquete estadístico para las ciencias sociales SPSS versión 14.0.

Variables: Como variables independientes se consideraron las siguientes: variables que componen el Clima Organizacional, los componentes de la Satisfacción Laboral y Variables Sociodemográficas. Mientras que como variables dependientes, se designaron los niveles de Agotamiento Emocional, Despersonalización y Realización Personal.

Objetivos: Se espera encontrar diferencias significativas entre hombres y mujeres en relación a las variables asociadas al síndrome de Burnout, a la percepción del clima y la satisfacción laboral.

Se espera explicar el surgimiento de las variables asociadas al síndrome de Burnout a través de las variables de clima laboral y satisfacción laboral diferenciando la muestra ente hombres y mujeres.

Instrumentos: Ver tabla 1. 


\section{Tabla 1: Instrumentos}

\begin{tabular}{|c|c|c|c|}
\hline Instrumento & Escalas & Definición & Confiabilidad \\
\hline \multirow{3}{*}{$\begin{array}{l}\text { Maslach } \\
\text { Bumout } \\
\text { Inventory. } \\
\text { Maslachy } \\
\text { Jackson, 1986. }\end{array}$} & Agotamiento Emocional. & \multirow{3}{*}{$\begin{array}{l}\text { Evalúa a través de } 22 \text { a fimaciones los sentimientos } \\
\text { personales y actitudes del profesional en el desempeño } \\
\text { de su trabajo. }\end{array}$} & \multirow{3}{*}{$\begin{array}{l}0.71 \text { a } 0.90 \\
\text { (Alarcón, Vazy } \\
\text { Guisado, 2002). }\end{array}$} \\
\hline & Despersonalización. & & \\
\hline & Realización Personal. & & \\
\hline \multirow{6}{*}{$\begin{array}{l}\text { Cuestionario } \\
\text { de } \\
\text { Satisfacción } \\
\text { Laboral } \\
\text { S21/26. } \\
\text { Meliá et als, } \\
1990 \text {. }\end{array}$} & $\begin{array}{l}\text { Satisfacción conla supervisión } \\
\text { y participación(SSP) }\end{array}$ & $\begin{array}{l}\text { Satisfacción con la supervisión de los superiores, } \\
\text { relación con los mismos, frecuencia de la supervisión, } \\
\text { justicia de trato recibido por la empresa, con la } \\
\text { formación recibida y con la participación en las } \\
\text { decisiones. }\end{array}$ & \multirow[t]{6}{*}{0.90} \\
\hline & $\begin{array}{l}\text { Satisfacción conlas } \\
\text { prestaciones básicas (SPB) }\end{array}$ & $\begin{array}{l}\text { Cumplimiento de convenios laborales, de incentivos } \\
\text { económicos, negociaciones laborales y salario, a la } \\
\text { promocióny a la formación. }\end{array}$ & \\
\hline & $\begin{array}{l}\text { Satisfacciónintrinseca del } \\
\text { trabajo(SIT) }\end{array}$ & $\begin{array}{l}\text { Satisfacciones que da el trabajo en si mismo, } \\
\text { oportunidades que ofrece el trabajo de hacer aquello que } \\
\text { gusta o en lo que se destaca y la capacidad para decidir } \\
\text { por uno mismo aspectos del trabajo. }\end{array}$ & \\
\hline & $\begin{array}{l}\text { Satisfacción con el ambiente } \\
\text { fisico (SAF) }\end{array}$ & $\begin{array}{l}\text { Relativos al entomo físico y al espacio en el lugar de } \\
\text { trabajo. }\end{array}$ & \\
\hline & $\begin{array}{l}\text { Satisfacción conla cantidady } \\
\text { producción en el trabajo } \\
\text { (SCP1) }\end{array}$ & $\begin{array}{l}\text { Ritmo y cantidad en que el trabajo se desarrolla y la } \\
\text { satisfacción que produce. }\end{array}$ & \\
\hline & $\begin{array}{l}\text { Satisfacción conla calidad de } \\
\text { producción en el trabajo } \\
\text { (SCP2) }\end{array}$ & $\begin{array}{l}\text { Evalúa los medios materiales de los que dispone el } \\
\text { trabajador para realizar su trabajo. }\end{array}$ & \\
\hline \multirow{8}{*}{$\begin{array}{l}\text { Escala de } \\
\text { Clima } \\
\text { Laboral. } \\
\text { Ramirez, } \\
2008 .\end{array}$} & Compromiso extrínseco (CE) & $\begin{array}{l}\text { Grado en que el trabajador evalúa el nivel de } \\
\text { vinculación de sus compañeros con el trabajo. }\end{array}$ & \multirow[t]{8}{*}{0.88} \\
\hline & Compañeros - cohesión (CC) & $\begin{array}{l}\text { Grado de cercanía y confianza, traducido en la } \\
\text { posibilidad de trabajar en equipo. }\end{array}$ & \\
\hline & Apoyo del supervisor (AS) & $\begin{array}{l}\text { Evalua ción sobre jefatura directa en relación al nivel de } \\
\text { soporte y cercania que brinda. }\end{array}$ & \\
\hline & Descripción de cargo (DC) & $\begin{array}{l}\text { Evaluación de la definición del cargo y conocimiento de } \\
\text { la descripción de este mismo. }\end{array}$ & \\
\hline & Infraestructura(IN) & $\begin{array}{l}\text { Evaluación de las condiciones y recursos físicos en } \\
\text { función de si permiten realizar de forma adecuada el } \\
\text { trabajo. }\end{array}$ & \\
\hline & $\begin{array}{l}\text { Equidady autonomia en el } \\
\text { desarrollo del trabajo(EA) }\end{array}$ & $\begin{array}{l}\text { Evaluación de aspectos que sugieren diferencias en el } \\
\text { trato de la organización hacia sus empleados y en la } \\
\text { libertad que éstos tienen para llevar a cabo la ejecución } \\
\text { de sus tareas. }\end{array}$ & \\
\hline & $\begin{array}{l}\text { Reconocimiento de méritos } \\
\text { (RM) }\end{array}$ & $\begin{array}{l}\text { Evaluación de la existencia de un sistema de valoración } \\
\text { del trabajo realizado a decuadamente, a demás de evaluar } \\
\text { la forma en que entrega dicho reconocimiento. }\end{array}$ & \\
\hline & Compromiso intrinseco $(\mathrm{CI})$ & $\begin{array}{l}\text { Sentimiento de pertenencia } 0 \text { identificación con la } \\
\text { organización y la intención de permanecer en ella. }\end{array}$ & \\
\hline $\begin{array}{l}\text { Cuestionario } \\
\text { Sociodemogrả } \\
\text { fico. }\end{array}$ & $\begin{array}{l}\text { Edad, sexo, nivel educacionaly } \\
\text { cargo desempeñado. }\end{array}$ & $\cdots$ & $\cdots$ \\
\hline
\end{tabular}

\section{Resultados}

De acuerdo a los análisis realizados, los resultados sugieren que existen diferencias estadísticamente significativas entre hombres y mujeres sólo en 5 variables del total estudiadas (tabla 2). Así, la escala del MBI, muestra que los hombres presentan un mayor puntaje de Despersonalización en relación a las mujeres. Por otro lado, con respecto al Clima Organizacional las mujeres presentarían un mayor Compromiso Intrínseco, tendencia que se mantiene en las variables que componen la Satisfacción Laboral, ya que las mujeres evaluadas evidenciarían una mayor Satisfacción con la Super- 
visión, Satisfacción con Remuneración y Satisfacción con la Calidad del Trabajo Producido, en comparación con los hombres.

Tabla 2: Diferencias de medias Hombres - Mujeres

\begin{tabular}{|c|c|c|c|c|c|c|}
\hline Variables & Sexo & $\mathbf{N}$ & Media & SD & $\mathbf{F}$ & $\mathbf{p}$ \\
\hline \multirow[t]{2}{*}{$\overline{\text { Despersonalización }\left({ }^{*}\right)}$} & Mujer & 100 & 6,9600 & 5,33129 & 12,573 & $\overline{, 001}$ \\
\hline & Hombre & 59 & 9,4746 & 6,84900 & & \\
\hline \multirow[t]{2}{*}{ Compromiso Intrínseco $(* *)$} & Mujer & 100 & 3,5400 & 51947 & 9,962 &, 002 \\
\hline & Hombre & 59 & 3,0932 &, 85957 & & \\
\hline \multirow[t]{2}{*}{ Satisfacción Supervisión $(* * *)$} & Mujer & 100 & 3,0580 & 53164 & 3,912 &, 050 \\
\hline & Hombre & 59 & 2,7841 &, 66827 & & \\
\hline \multirow[t]{2}{*}{ Satisfacción Remuneración (***) } & Mujer & 100 & 2,6180 & 54762 & 5,705 & $\overline{, 018}$ \\
\hline & Hombre & 59 & 2,5222 &, 72004 & & \\
\hline \multirow[t]{2}{*}{ Satisfacción Calidad $(* * *)$} & Mujer & 100 & 2,9500 & 61751 & 3,894 &, 050 \\
\hline & Hombre & 59 & 2,8051 &, 72526 & & \\
\hline
\end{tabular}

(*) Maslach Burnout Inventory.

(**) Escala de Clima Organizacional.

$(* * *)$ Cuestionario de Satisfacción Laboral S21/26

Al realizar un análisis de los aspectos que podrían explicar el surgimiento de las variables del síndrome de Burnout, los resultados sugieren que para la muestra de mujeres (tabla 3), los altos indicadores de los sentimientos de Agotamiento Emocional, se explicarían por los bajos niveles de Satisfacción Intrínseca y Satisfacción con la Calidad del Trabajo $\left(\mathbf{R}^{2}=\mathbf{1 2 4}\right)$. Los altos sentimientos de Despersonalización se explicarían por la baja Satisfacción Intrínseca y los bajos niveles de Satisfacción con el Ambiente $\left(\mathbf{R}^{2}=\mathbf{1 6 5}\right)$. Por el contrario, los elevados sentimientos de Realización Personal se podrían explicar por la Satisfacción con la Remuneración y la adecuada Descripción de Cargo $\left(\mathbf{R}^{2}=\mathbf{, 1 9 5}\right)$.

\section{Tabla 3: Análisis de regresión V.D Variables de MBI - V.I Variables Clima Organizacional - Satisfacción Laboral MUJERES}

\begin{tabular}{l|l|l|l|l|r|l}
\hline Modelo & Variables Independientes & \multicolumn{1}{|c|}{$\begin{array}{c}\text { Variables } \\
\text { Dependientes }\end{array}$} & $\mathbf{F}$ & $\mathbf{t}$ & $\mathbf{R}^{\mathbf{2}}$ Ajustada & Sig. \\
\hline $\mathbf{1}$ & $\begin{array}{l}\text { Satisfacción Intrínseca - } \\
\text { Satisfacción Calidad }\end{array}$ & $\begin{array}{l}\text { Agotamiento } \\
\text { Emocional }\end{array}$ & 9,999 & $-2,034$ & $\mathbf{, 1 2 4}$ &, $000 \mathrm{~b}$ \\
\hline $\mathbf{1}$ & $\begin{array}{l}\text { Satisfacción Intrínseca - } \\
\text { Satisfacción Ambiente }\end{array}$ & Despersonalización & 10,754 & $-1,987$ &, $\mathbf{1 6 5}$ &, $000 \mathrm{~b}$ \\
\hline $\mathbf{1}$ & $\begin{array}{l}\text { Satisfacción Remuneración } \\
\text { - Descripción Cargo }\end{array}$ & Realización Personal & 16,399 & 3,006 &, $\mathbf{1 9 5}$ &, $000 \mathrm{~b} *$ \\
\hline
\end{tabular}

$* * \mathrm{p}<.05$ 
Por otro lado, al realizar el mismo análisis, pero ahora con la muestra sólo de hombres (tabla 4) los resultados sugieren que los aspectos que explicarían el surgimiento de las variables del síndrome de Burnout relacionan la baja percepción de Equidad - Autonomía a los sentimientos de Agotamiento Emocional $\left(\mathbf{R}^{2}=\mathbf{4 3 1}\right)$ que los trabajadores experimentan. Los sentimientos de Despersonalización experimentados podrían explicarse por la baja percepción de Compromiso Extrínseco referido al bajo nivel de vinculación de sus compañeros con el trabajo $\left(\mathbf{R}^{2}=\mathbf{, 1 7 1}\right)$. En cambio los altos niveles de Realización Personal se encuentran explicados por el Compromiso Intrínseco que los trabajadores evaluados sienten $\left(\mathbf{R}^{2}=\mathbf{1}, \mathbf{7 4}\right)$.

\section{Tabla 4: Análisis de regresión V.D Variables de MBI - V.I Variables Clima Organizacional - Satisfacción Laboral HOMBRES}

\begin{tabular}{l|l|l|c|c|c|c}
\hline Modelo & Variables Independientes & \multicolumn{1}{|c|}{$\begin{array}{c}\text { Variables } \\
\text { Dependientes }\end{array}$} & $\mathbf{F}$ & $\mathbf{t}$ & $\mathbf{R}^{2}$ Ajustada & Sig. \\
\hline $\mathbf{1}$ & Equidad Autonomía & $\begin{array}{l}\text { Agotamiento } \\
\text { Emocional }\end{array}$ & 55,605 & $-7,457$ & $\mathbf{, 4 3 1}$ &, $000 \mathrm{a}$ \\
\hline $\mathbf{1}$ & Compromiso Extrinseco & Despersonalización & 12,957 & $-3,600$ & $\mathbf{, 1 7 1}$ &, $001 \mathrm{a}$ \\
\hline $\mathbf{1}$ & Compromiso Intrínseco & Realización Personal & 16,190 & 4,024 & $\mathbf{, 1 7 4}$ &, $000 \mathrm{a}$ \\
\hline
\end{tabular}

$* * \mathrm{p}<.05$

\section{Discusiones y conclusiones}

Se podría afirmar, de acuerdo a los objetivos planteados y a los resultados obtenidos, que los hombres que se desempeñan en puestos de trabajo asociados tanto a la educación, la salud, el comercio de tangibles e intangibles y a la administración pública de la ciudad de Arica, presentan mayores niveles de despersonalización que las mujeres. La despersonalización es una de las variables asociadas al síndrome de Burnout que se caracteriza por el desarrollo de sentimientos, actitudes y respuestas negativas, distantes y frías hacia otras personas, especialmente hacia los beneficiarios del propio trabajo. Para el resto de las variables estudiadas son las mujeres dedicadas a estas mismas actividades laborales las que lideran los puntajes significativos en relación a los obtenidos por los hombres. Es decir, con respecto al clima laboral, son las mujeres las que evidencian sentimientos de pertenencia o identificación con la organización en la cual trabajan, además de mantener la clara intención de permanecer en ella. Además, en relación a la satisfacción laboral, son las mujeres las que presentan mayor satisfacción con la supervisión de sus superiores, la 
relación que mantienen con ellos mismos, la frecuencia de la supervisión, la percepción de justicia del trato recibido por la empresa, con la formación recibida y con la participación en las decisiones. Evidencian también sentirse más satisfechas que los hombres en relación al cumplimiento de convenios laborales, de incentivos económicos, negociaciones laborales, salarios, promoción y a la formación, evaluando así de mejor manera los medios materiales de los que disponen para realizar su trabajo.

Es interesante observar estas diferencias, ya que se mantienen también en el surgimiento de las variables asociadas al síndrome de Burnout. Por lo que hombres y mujeres presentarían distintas razones que gatillarían las distintas variables del Síndrome, lo que implicaría diferentes formas de abordamiento y prevención de este al interior de los puestos de trabajo.

Es así que para las mujeres sentir un alto agotamiento o cansancio emocional, es decir, sentir una fatiga que se manifiesta física o psíquicamente mezclado con una sensación de no poder dar más de así mismas a los demás se explicaría por un baja satisfacción en el trabajo en sí mismo, el percibir pocas oportunidades para hacer aquello que realmente les gusta, aquello en lo que destaca, o sentirse limitada para tomar decisiones, así como también se explicaría por una baja evaluación de los medios materiales de los que disponen para realizar su trabajo. Y para los hombres, este mismo nivel elevado de agotamiento emocional lo explicaría la baja percepción de equidad y autonomía, es decir, lo que provocaría en los hombres la fatiga física o psíquica serían las diferencias en el trato de la organización hacia sus empleados y la percepción de una baja libertad que éstos tienen para llevar a cabo la ejecución de sus tareas, resultados que concuerdan con los propuesto por Robbins (1999).

Por otro lado, el desarrollar sentimientos, actitudes y respuestas negativas distantes y frías hacia otras personas, especialmente hacia los beneficiarios del propio trabajo (despersonalización), surgiría en las mujeres por sentir que no tienen oportunidades laborales, posibilidades de hacer lo que les gusta o tomar decisiones, así como también por una baja satisfacción con su entorno físico y espacio en el lugar de trabajo. A diferencia de los hombres, la alta despersonalización se explicaría por el nivel de vinculación de sus compañeros con el trabajo.

La aparición de una alta realización personal, caracterizada por el sentido de la propia vida orientado a los logros personales y una adecuada autoestima, en las mujeres se ve explicado por la satisfacción que éstas sienten hacia las prestaciones básicas y una claridad en la descripción de sus responsabilidades como trabajadoras, es decir, presentan altos niveles de realización personal en la medida que obtengan el cumplimiento de sus convenios laborales, sueldo, incentivos económicos, negociaciones, promociones, etc, así como también mientras exista una clara definición del cargo que desempeñan y conozcan la descripción de este mismo. Mientras que los hombres, el tener altos niveles de realización personal se explica 
por el sentimiento de pertenencia o identificación con la organización en la que trabajan y su intención de permanecer en ella.

Por lo tanto, se han encontrado diferencias significativas entre hombres y mujeres, tanto en las diferencias de medias que arrojaron los resultados de los instrumentos que miden el síndrome de Burnout, el clima y la satisfacción laboral, así como también presentaron diferencias significativas en los resultados que explicarían el surgimiento de las variables asociadas a este mismo Síndrome.

Es interesante tener en cuenta estas diferencias, ya que la importancia que ha cobrado el trabajo para la vida de las personas ha obligado a las organizaciones y a la sociedad en general prestar mayor atención a este ámbito para velar por la seguridad y el bienestar de los trabajadores tanto en términos físicos como psicológicos (Peiró y Bravo, 1999; Ramírez, 2009). Y el prestar atención sugiere poseer las herramientas adecuadas para una intervención directa y efectiva.

Con los resultados del presente estudio, se obtiene valiosa información para crear dentro de las organizaciones los factores protectores correspondientes, tanto para hombres como para mujeres, para apaliar los efectos del surgimiento de las variables asociadas al síndrome de Burnout, tomando en cuenta que, según Martín, Luceño, Jaén y Rubio (2007), los riesgos psicosociales en el entorno laboral se relacionan directamente con enfermedades.

Es interesante destacar que con respecto al clima y la satisfacción laboral, las diferencias de medias que resultaron ser significativas entre hombres y mujeres, evidencian mayor satisfacción de las mujeres sobre los hombres, por lo que se sugiere para futuras investigaciones estudiar, desde una perspectiva de género, los factores que podrían estar influyendo en estos interesantes resultados.

Como limitación de este estudio se encuentra una baja cantidad cuestionarios demográficos completados de forma correcta, que redujo la muestra total, ya que los incompletos no fueron considerados para los resultados finales. Esto se puede explicar por el temor de los trabajadores de identificarse, a pesar de la confidencialidad y anonimato de sus respuestas, aspecto que es necesario mejorar para ampliar las posibilidades de respuesta y abarcar a un conglomerado mayor de trabajdores, favoreciendo el desarrollo del conocimiento. 
Polis, Revista de la Universidad Bolivariana, Volumen 10, $N^{\circ}$ 30, 2011

\section{Nota}

${ }^{1}$ Investigación presentada en el V Encuentro Iberoamericano de Investigación en Salud Ocupacional (EIISO), Universidad Católica del Maule, Talca, Chile. 22-23 de octubre, año 2009. 


\section{Bibliografía}

Alarcón, J., Vaz, F. y Guisado, J. (2002), “Análisis del síndrome de burnout: psicopatología, estilos de afrontamiento y clima social (II)”, en Revista de Psiquiatría de la Facultad de Medicina Barna. Vol. 29, nº1, pp. 8 - 17.

Chiang, M., Salazar, C. y Núñez, A. (2007), “Clima organizacional y satisfacción laboral en un establecimiento de salud estatal: Hospital tipo 1”, en Theoria. Vol. 16, $\mathrm{n}^{\circ} 2$, pp. $61-76$.

Chomali, F. y Marjuf, N. (2007), Ética y responsabilidad social en la empresa. $2^{\circ}$ Edición. Aguilar Chilena de Ediciones S.A.

Demerouti, E., Bakker, A., De Jonge, J. y Schaufeli, W. B. (2001), “Burnout and engagement at work as function of demands and control”, en Scand $J$ Work Environ Health. Vol. 27, n 27, pp. 279 - 286.

Diego, R., Diego, J. y Olivar, S. (2001), “Job satisfaction in banking workers”, en Psicothema. Vol. 13, n 4, pp. 629 - 635.

Dormann, C., Fay, D., Zapf, D. y Frese, M. (2006), “A State-Trait Analysis of Job Satisfaction: On the Effect of Core Self-Evaluations”, en Applied psychology: an international review. Vol. 55, $\mathrm{n}^{\circ} 1$, pp. $27-51$.

Duran, M., Extremera, N., Montalbán, F. y Rey, L. (2005), “Engagement y Burnout en el ámbito docente: Análisis de sus relaciones con la satisfacción laboral y vital en una muestra de profesores”, en Revista de Psicología del Trabajo y de las Organizaciones. Vol. 21, $\mathrm{n}^{0}$ 1-2, pp. 145 - 158.

Extremera, N., Durán, A. y Rey, L. (2007), “Inteligencia emocional y su relación con los niveles de burnout, engagement y estrés en estudiantes universitarios”, en Revista de Educación. Vol. 342, Enero-Abril, pp. $239-256$.

Fernández, B., y Paravic, T. (2007), “Nivel de satisfacción laboral en enfermeras de hospitales públicos y privados de la provincia de Concepción, Chile”, en Ciencia y Enfermeria. Vol. 2, nº 9, pp. 57-66.

Gil - Monte, P. y Peiró, J. (1999), “Perspectivas teóricas y modelos interpretativos para el estudio del síndrome de quemarse por el trabajo”, en Anales de Psicología. Vol. 15, n 2, pp. 261 - 268.

Gomes, C. y Elizalde R. (2009), “Trabajo, tiempo libre y ocio en la contemporaneidad: Contradicciones y desafíos”, en Polis, Revista de la Universidad Bolivariana. Vol. 8, n²2, pp. 249-266.

González, A. y Parera, I. (2005), “Clima Organizacional: Resultados del diagnóstico en una empresa”, en Revista Transporte, Desarrollo y Medio 
Ambiente. Vol. 25. $\mathrm{n}^{\circ}$ 1, pp. $42-44$.

Instituto Nacional de Seguridad e Higiene en el Trabajo, INSHT. (1997). II Encuesta Nacional de Condiciones de Trabajo. Barcelona: INSHT.

Italia, S., Favara-Scacco, C., Di Cataldo, A. y Russo, G. (2008), "Evaluation and art therapy treatment of the burnout syndrome in oncology units", en Psycho - Oncology. 2008. Vol. 17, pp. 676 - 680.

Laros, J. y Puente-Palacios, K. (2004), "Validaçao cruzada de uma escala de Clima Organizacional”, en Estudios de Psicología. Vol. 9. n 1, pp. 113 -119 .

Leiter, M.P. y Schaufeli, W. B. (1996), “Consistency of the burnout construct across occupations”, en Anxiety, Stress and Copyng. Vol. 9, pp. 229 - 243.

Lisbona, A., Palací, F. y Gómez, A. (2008), “Escala de clima para la iniciativa y para la seguridad psicológica: adaptación al castellano y su relación con el desempeño organizacional”, en Revista de Psicología del Trabajo y las Organizaciones. Vol. 24. $\mathrm{n}^{\mathrm{0}}$ 2, pp. $153-167$.

Martín, J., Luceño, L., Jaén, M. y Rubio, S. (2007), “Relación entre factores psicosociales adversos, evaluados a través del cuestionario multidimensional Decore, y salud laboral deficiente”, en Psicothema. Vol. 19, no 1, pp. $95-101$.

Maslach, C. y Jackson, S.E. (1986). MBI (2 Ed., $1^{\circ}$ Ed. De 1981). Palo Alto, California: Consulting Psychologist Press.

Maslach, C., Schaufeli, W. B. y Leiter, M. (2001), “Job Burnout”, en Annual Review of Psychology. Vol. 52, pp. 397 - 422.

Meliá, J.L., y Peiró, J. M. (1989), “El Cuestionario de Satisfacción S10/ 12: Estructura factorial, fiabilidad y validez”, en Revista de Psicología del Trabajo y de las Organizaciones. Vol. 4, nº 11, pp. 179 - 187.

Meliá, J., Pradilla, J., Martí, N., Sancerni, M., Oliver, A. y Tomas, J. (1990), "Estructura factorial, fiabilidad y validez del cuestionario de satisfacción S21/26: Un instrumento con formato dicotómico orientado al trabajo profesional”, en Revista de Psicología Universitas Tarraconensis. Vol. 12, $\mathrm{n}^{\circ}$ 1-2, pp. $25-39$.

Moreno-Jiménez, B., Seminotti, R., Garrosa, E., Rodríguez-Carvajal, R. y Morante, M. (2005), "El Burnout médico: la ansiedad y los procesos de afrontamiento como factores intervinientes”, en Ansiedad y Estrés. Vol. $11, \mathrm{n}^{\circ} 1$, pp. $180-194$.

Muñoz, H., Guerra de los Santos, J., Barón, M. y Munduate, L. (2006), “El acoso psicológico desde una perspectiva organizacional. Papel del clima 
organizacional y los procesos de cambio”, en Revista de Psicología del Trabajo y las Organizaciones. Vol. 22. n 3, pp. 347 - 361.

Oliver, A., Tomas, J. M. y Cheyne, A. (2005), “Clima de seguridad laboral: naturaleza y poder predictivo”, en Revista de psicología del trabajo y de las organizaciones. Vol. 21, n 3, pp. $253-268$.

Ortega Ruiz, C. y López Ríos, F. (2004), “El Burnout o síndrome de estar quemado en los profesionales sanitarios: revisión y perspectivas”, en International Journal of Clinical and Health Psychology. Vol. 4. $\mathrm{n}^{\mathrm{o}} 1$, pp. 137 - 160 .

Peiró, J. (1999), "Valoración de riesgos psicosociales y estrategias de prevención: el modelo “AMIGO” como base de la metodología Prevenlab/ Psicosocial”, en Revista de Psicología del Trabajo y las Organizaciones. Vol.15, no 2, pp. $267-314$.

Peiró, J. y Bravo, M. (1999), “Factores psicosociales en la prevención de riesgos laborales: oportunidades y retos para la Psicología del Trabajo y de las Organizaciones”, en Revista de Psicología del trabajo y las organizaciones. Vol. 15. $\mathrm{n}^{\circ} 2$, pp. 137-146.

Pozo, C., Alonso, E., Hernández, S. y Martos, Mª J. (2005), “Determinantes de la satisfacción laboral en trabajadores de la administración pública: el valor de las relaciones interpersonales en el lugar de trabajo”, en Ansiedad y Estrés. Vol. 11 n 2 - 3, pp. 247 - 264.

Ramírez, M. (2009). Análisis de Variables Organizacionales y Variables Individuales para el surgimiento de Satisfacción Laboral, Síndrome de Burnout, Síndrome de Estrés y Engagement dentro del Contexto Organizacional. Tesis Master en Prevención de Riesgos. Universitat Politècnica de Catalunya, 2009.

Ramírez, M. y Zurita, R. (2009), “Variables organizacionales y psicosociales asociadas al síndrome de burnout en trabajadores del ámbito educacional” en Polis, Revistas de la Universidad Bolivariana. Vol. 9, $\mathrm{n}^{\circ}$ 25, pp.537553.

Robbins, S. (1999), Comportamiento Organizacional, $8^{\circ}$ Edición. Prentice Hall.

Salgado, J., Remeseiro, C. e Iglesias, M. (1996)., “Clima organizacional y satisfacción laboral en una PYME”, en Psicothema. Vol. 8. n 2, pp. $329-$ 335.

Sánchez, S., Guillén, C. y León, M. (2006), “La autoeficacia percibida en el afrontamiento de riesgos laborales psicosociales como variable moduladora del burnout”, en Ansiedad y Estrés. Vol. 12, n 2 - 3, pp.495 504. 
Zohar, D. y Luria, G. (2004), "Climate as a social-cognitive construction of supervisory safety practices: scripts as proxy of behavior patterns", en Journal of Applied Psychology. Vol. 89 (2), 322-333.

Recibido: 11.12.2009

Aceptado: 14.09.2011 